\title{
UPTHROW OF OBJECTS DUE TO HORIZONTAL IMPULSE EXCITATION
}

\author{
By Ioannis N. Psycharis and Paul C. Jennings
}

\begin{abstract}
The phenomenon of upthrow of rigid blocks, excited into motion by horizontal impulses, is examined in this study. A simple foundation model is used, consisting of two sets of springs and dashpots; one set near each corner of the block. Each set consists of vertical and horizontal elements. The vertical springs are not permitted to take tensile forces, so separation of the block from the ground is allowed. The associated horizontal spring separates along with the vertical spring. The response can be separated into three regimes, namely, full contact, partial uplift, and complete separation. Analytical expressions for the response are given for the first two cases; the third case is simply rigid body motion. The overall response is nonlinear and analytical criteria for upthrow were not found. The minimum value of the impulse required for complete separation, expressed as a normalized initial velocity, was determined numerically and a parametric investigation performed. Although the irregularity of the results does not allow many general conclusions to be drawn, it was found that for short blocks, a decrease in the stiffness of the horizontal foundation springs required larger impulses for separation. These effects had mixed results for tall blocks.
\end{abstract}

\section{INTRODUCTION}

The phenomenon of complete separation of objects from their supports has been among the notable effects of many major earthquakes. The observations are usually restricted to the areas of strongest shaking and range from evidence of stones being tossed into the air in the Great Assam earthquake of 1897 (Oldham, 1899) to indication of large vertical motions of a 20-ton fire truck and its shed (Morrill, 1971) in the San Fernando earthquake of 1971. Observations of this type of behavior have been widely reported, e.g., both in Japan (Matsuzawa, 1944; Ikegami and Kishinouye, 1950; Kawasumi, 1950) and in California (Lawson, 1908; Richter, 1958; Clark, 1972). For a historical review of the subject, the reader is referred to the excellent summary of significant observations presented by Bolt and Hansen (1977). Attention is directed also to the work by Ishiyama $(1980,1982)$.

Objects can be separated from their foundations by a variety of mechanisms, including vertical motion, slipping, and rocking, acting singly or in combination. The vertical separation that can result is called upthrow, a term used in this study. This terminology arises from the simplest mechanism by which separation occurs, a tossing into the air. In many cases, this is the mechanism observers believed responsible for separation, and this conclusion is commonly phrased as "evidence of vertical ground accelerations greater than gravity." This conclusion is inaccurate and somewhat misleading. It is misleading in the sense that acceleration of a rigid foundation upwards in excess of gravity does not cause separation of a supported object, acceleration downward of the foundation with an absolute value greater than gravity is what is required. In the case of a stone resting on rigid ground this means that the ground must at some time accelerate downward faster than a falling body, which requires tension in the foundation.

More important, the conclusion is inaccurate because, as several investigators have noted, separation is achievable by other, more realistic mechanisms which 
require lower accelerations. For example, Newmark (1973) showed that separation of an object from the ground is possible for much smaller vertical accelerations if the foundation is assumed to be elastic. The analysis of Newmark was taken even further by Bolt and Hansen (1977) who worked out in detail two mechanics problems, showing that vertical separation of components of mechanical systems can occur without the downward ground acceleration exceeding the acceleration of gravity. In fact, as the complexity of the foundation is increased, vertical separation can occur in the complete absence of vertical excitation. In a recent investigation of the rocking behavior of rigid blocks supported by an elastic foundation which is not allowed to take tensile forces, Psycharis and Jennings (1982) showed that complete separation of the block from the foundation is possible even for purely

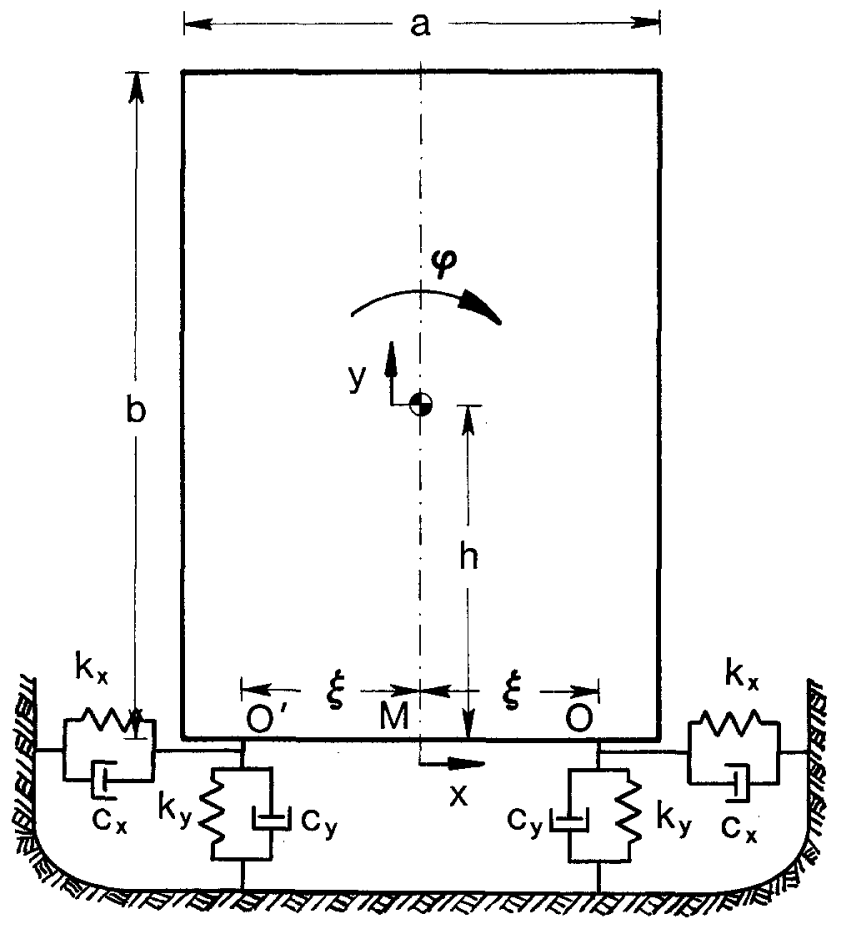

FIG. 1. A rigid block on a compliant foundation. Partial separation occurs when the vertical deflection of $O$ or $O^{\prime}$ exceeds the static deflection of the vertical spring. The block has an initial horizontal velocity, $v_{0}$, caused by an impulsive motion of the base.

horizontal excitations. In this analysis, the block was constrained against horizontal movement at its base and, thus, sliding was prevented and only rocking and vertical motions were permitted. An interesting result was that for very short blocks, complete separation is possible for any excitation strong enough to cause partial uplift.

The present study examines the upthrow of a rigid block supported by a foundation with both horizontal and vertical flexibility. The system considered is shown in Figure 1. It consists of a rigid block of mass $m$ supported by vertical and horizontal springs and dashpots of constants $k_{y}, k_{x}, c_{y}$ and $c_{x}$, respectively, symmetrically placed under the base. For the limiting case of $k_{x} \rightarrow \infty$ (i.e., the base of the block is not allowed to move horizontally on its foundation), it has been shown by Psycharis and Jennings (1982) that the two-spring foundation can satisfactorily 
model the more realistic, but more complex, Winkler foundation. Although it has not been examined whether this assumption can be extended to the case in which horizontal oscillations are also considered, it is believed that the two-spring foundation, used here because of its simplicity, does model some of the essential features of a flexible foundation that permits lift-off.

It is assumed that the vertical springs cannot take tensile forces, therefore, separation occurs whenever the upward displacement of point $O$ or $O^{\prime}$ exceeds the static deflection, $\delta$. When this occurs, separation from the horizontal spring is assumed to take place also. Under these assumptions, the vertical spring is unstrained at the time of uplift, while the horizontal one will, in general, be under load. It is assumed in the analysis that this spring returns to its equilibrium position immediately after separation.

The response of the block, initially at rest, is examined for a base excitation consisting of a horizontal impulse of magnitude $I$. This is equivalent to examining the free vibrations of the block with an initial horizontal velocity, $v_{0}=I / m$, mounted on an immovable base. This excitation was chosen because of its simplicity; it allows a better understanding of the dynamic behavior of the system than more complex excitation, and captures some of the dynamic features of the earthquake problem. Since the aim of this study is to examine the parameters that affect complete separation of the block from its foundation, the minimum required value of $v_{0}$ for upthrow to occur is determined and its dependence on the parameters of the system is examined in the following analysis. Vertical excitations were not considered; for the symmetric block in Figure 1, the problem of undamped vertical excitation has been treated by Newmark (1973).

\section{EquATIONS OF Motion}

The system, as defined in the introduction and shown in Figure 1, possesses three degrees of freedom, which are taken for convenience as vertical translation measured by the vertical displacement, $y$, of the center of mass; horizontal translation measured by the horizontal displacement, $x$, of the mid-point of the base, $M$; and rotation in the plane of motion measured by the angle of tilting, $\varphi$, from the vertical. There are two sets of equations governing the motion of the block before complete separation, corresponding to the two regimes of the response: during full contact, when both springs are in contact with the block, and after partial uplift, when only one spring is in contact. For the case of free oscillations, the linearized equations of motion for small displacement can be written as follows

FULL CONTACT

$$
\begin{gathered}
\dot{x}+h \ddot{\varphi}+2 \zeta_{x} \omega_{x} \dot{x}+\omega_{x}^{2} x=0 \\
h \ddot{\varphi}+\frac{\lambda}{\alpha^{2}} \ddot{x}+2 \lambda \zeta_{y} \omega_{y} h \dot{\varphi}+\lambda \omega_{x}^{2} h \varphi=0 \\
\ddot{y}+2 \zeta_{y} \omega_{y} \dot{y}+\omega_{y}^{2} y=0
\end{gathered}
$$

AFTER PARTIAL UPLIFT

$$
\ddot{x}+h \ddot{\varphi}+\zeta_{x} \omega_{x} \dot{x}+\frac{1}{2} \omega_{x}^{2} x=0
$$




$$
\begin{gathered}
h \ddot{\varphi}+\frac{\lambda}{\alpha^{2}} \ddot{x}+\lambda \zeta_{y} \omega_{y} h \dot{\varphi}+\frac{\lambda}{\alpha} \zeta_{y} \omega_{y} \dot{y}+\frac{\lambda}{2} \omega_{y}^{2} h \varphi+\frac{\lambda}{2 \alpha} \omega_{y}^{2} y= \pm \frac{\lambda g}{2 \alpha} \\
\ddot{y}+\zeta_{y} \omega_{y} \dot{y} \pm \alpha \zeta_{y} \omega_{y} h \dot{\varphi}+\frac{1}{2} \omega_{y}^{2} y \pm \frac{\alpha}{2} \omega_{y}^{2} h \varphi=-\frac{g}{2}
\end{gathered}
$$

where,

$$
\begin{aligned}
\omega_{x} & =\sqrt{\frac{2 k_{x}}{m}} \\
\omega_{y} & =\sqrt{\frac{2 k_{y}}{m}} \\
\zeta_{x} & =\frac{c_{x}}{m \omega_{x}} \\
\zeta_{y} & =\frac{c_{y}}{m \omega_{y}} \\
\alpha & =\xi / h \\
\lambda & =\frac{m \xi^{2}}{I_{M}} .
\end{aligned}
$$

In equation (12), $I_{M}$ is the moment of inertia about the mid-point of the base, $M$. Whenever a double sign appears in the equations of motion after lift-off, the upper sign corresponds to positive angles of rotation and the lower one to negative angles. Also, small gravity terms which would appear in some of the restoring terms in the left-hand side of these equations were neglected. For example, the exact equation corresponding to equation (2) is

$$
h \ddot{\varphi}+\frac{\lambda}{\alpha^{2}} \ddot{x}+2 \lambda \zeta_{y} \omega_{y} h \dot{\varphi}+\left(\frac{2 k_{y} \xi^{2}-m g h}{I_{M}}\right) h \varphi=0 .
$$

However, for realistic values of soil stiffness, the term $2 k_{y} \xi^{2}$ is much greater than the term $m g h$, which can be neglected. The same approximation was made in equation (5).

The characteristic frequencies $\omega_{x}$ and $\omega_{y}$ which appear in the above equations are the frequencies of purely horizontal and purely vertical motions, respectively. The third eigenfrequency, $\omega_{\varphi}$, related to rocking motion about the center of the base, does not appear explicitly, because it can be expressed in terms of $\omega_{y}$ under the assumptions made. More specifically,

$$
\omega_{\varphi}^{2}=\frac{2 k_{y} \xi^{2}}{I_{M}}
$$

and using (8) and (12)

$$
\omega_{\varphi}=\sqrt{\lambda} \omega_{y} .
$$


A similar expression holds for the ratio of critical damping, $\zeta_{\varphi}$, in rocking, which can be written as

$$
\zeta_{\varphi}=\sqrt{\lambda} \zeta_{y} .
$$

The equation describing the vertical motion of the block during full contact is uncoupled from the rocking and the horizontal movement; as a consequence, vertical vibrations are not excited initially for purely horizontal excitation. However, after partial uplift occurs, all the equations are coupled and vertical oscillations begin. The block will continue to vibrate in all three modes thereafter.

The solution of the equations of motion during full contact can be written as

$$
\begin{aligned}
h \varphi(t)=\left(A_{1} \sin \tilde{\omega}_{1 d} t\right. & \left.+A_{2} \cos \tilde{\omega}_{1 d} t\right) e^{-\tilde{\xi}_{1} \tilde{\omega}_{1} t}+\left(A_{3} \sin \tilde{\omega}_{2 d} t+A_{4} \cos \tilde{\omega}_{2 d} t\right) e^{-\tilde{\zeta}_{2} \tilde{\omega}_{2} t} \\
x(t)= & \left(\frac{\tilde{\omega}_{1}{ }^{2}}{\omega_{x}{ }^{2}-\tilde{\omega}_{1}{ }^{2}}\right)\left(A_{1} \sin \tilde{\omega}_{1 d} t+A_{2} \cos \tilde{\omega}_{1 d} t\right) e^{-\tilde{\zeta}_{1} \tilde{\omega}_{1} t} \\
& +\left(\frac{\tilde{\omega}_{2}{ }^{2}}{\omega_{x}{ }^{2}-\tilde{\omega}_{2}{ }^{2}}\right)\left(A_{3} \sin \tilde{\omega}_{2 d} t+A_{4} \cos \tilde{\omega}_{2 d} t\right) e^{-\tilde{\delta}_{2} \tilde{\omega}_{2} t} \\
& y(t)=\left(A_{5} \sin \omega_{y d} t+A_{6} \cos \omega_{y d} t\right) e^{-\zeta_{y} \omega_{y} t}
\end{aligned}
$$

in which,

$$
\begin{gathered}
\tilde{\omega}_{1,2}^{2}=\frac{\omega_{x}^{2}+\lambda \omega_{y}^{2} \pm \sqrt{\left(\omega_{x}{ }^{2}-\lambda \omega_{y}{ }^{2}\right)^{2}+\frac{4 \lambda^{2}}{\alpha^{2}} \omega_{x}{ }^{2} \omega_{y}{ }^{2}}}{2\left(1-\frac{\lambda}{\alpha^{2}}\right)} \\
\tilde{\zeta}_{i}=\frac{\zeta_{x}\left(\frac{\tilde{\omega}_{i}}{\omega_{y}}-\frac{\lambda \omega_{y}}{\tilde{\omega}_{i}}\right)+\lambda \zeta_{y}\left(\frac{\tilde{\omega}_{i}}{\omega_{x}}-\frac{\omega_{x}}{\tilde{\omega}_{i}}\right)}{2\left(1-\frac{\lambda}{\alpha^{2}}\right) \frac{\tilde{\omega}_{i}^{2}}{\omega_{x} \omega_{y}}-\frac{\omega_{x}}{\omega_{y}}-\frac{\lambda \omega_{y}}{\omega_{x}}}, \quad i=1,2 \\
\tilde{\omega}_{i d}=\tilde{\omega}_{i} \sqrt{1-\tilde{\zeta}_{i}^{2}}, \quad i=1,2 \\
\omega_{y d}=\omega_{y} \sqrt{1-\zeta_{y}^{2}}
\end{gathered}
$$

and the coefficients $A_{1}$ to $A_{6}$ will be determined by the initial conditions. It should be noted that equations (16) and (17) are correct to first order in the $\zeta$ 's only; thus, the damping factors cannot be too large.

For a horizontal impulse excitation, all the initial conditions are zero, except that $\dot{x}(0)=v_{0}=I / m$, where $I$ is the magnitude of the impulse. In this case

$$
A_{2}=A_{4}=A_{5}=A_{6}=0
$$


and

$$
\begin{aligned}
& A_{1}=\frac{v_{0}}{\tilde{\omega}_{1 d}} \frac{\left(\omega_{x}^{2}-\tilde{\omega}_{1}^{2}\right)\left(\omega_{x}^{2}-\tilde{\omega}_{2}^{2}\right)}{\omega_{x}^{2}\left(\tilde{\omega}_{1}^{2}-\tilde{\omega}_{2}^{2}\right)} \\
& A_{3}=\frac{v_{0}}{\tilde{\omega}_{2 d}} \frac{\left(\omega_{x}^{2}-\tilde{\omega}_{1}^{2}\right)\left(\omega_{x}^{2}-\tilde{\omega}_{2}^{2}\right)}{\omega_{x}^{2}\left(\tilde{\omega}_{2}^{2}-\tilde{\omega}_{1}^{2}\right)} .
\end{aligned}
$$

Since $y=0$, partial separation will occur when $\varphi$ reaches the critical angle, $\varphi_{c r}$, defined by

$$
h \varphi_{c r}=h \cdot \frac{\delta}{\xi}=\frac{g}{\alpha \omega_{y}^{2}}
$$

$\delta$ being the static deflection.

Without loss of generality, we will assume that $\varphi>0$. Then, the equations of motion after partial uplift can be rewritten in the form

$$
\begin{gathered}
\ddot{x}+\frac{\alpha \lambda}{\alpha^{2}(1+\lambda)-\lambda} \ddot{Y}+\frac{\alpha^{2}(1+\lambda)}{\alpha^{2}(1+\lambda)-\lambda} \zeta_{x} \omega_{x} \dot{x}+\frac{\alpha^{2}(1+\lambda)}{2\left[\alpha^{2}(1+\lambda)-\lambda\right]} \omega_{x}{ }^{2} x \\
=\frac{\alpha \lambda g}{\alpha^{2}(1+\lambda)-\lambda} \\
\ddot{Y}+\frac{\lambda}{\alpha} \ddot{x}+(1+\lambda) \zeta_{y} \omega_{y} \dot{Y}+\frac{1+\lambda}{2} \omega_{y}{ }^{2} Y=\frac{(1-\lambda) g}{2} \\
h \ddot{\varphi}=-\frac{\lambda g}{\alpha(1+\lambda)}-\frac{\lambda}{\alpha^{2}(1+\lambda)} \ddot{x}+\frac{\lambda}{\alpha(1+\lambda)} \ddot{Y}
\end{gathered}
$$

where $Y$ is defined by the equation

$$
Y=\zeta \varphi-y
$$

and denotes the vertical displacement, positive downwards, of the point of contact, $O$. Equations (21) and (22) do not depend explicitly on $\varphi$, and their solution can be written as

$$
\begin{aligned}
Y(t)= & \left(B_{1} \sin \tilde{\Omega}_{1 d} t+B_{2} \cos \tilde{\Omega}_{1 d} t\right) e^{-\tilde{Z}_{1} \tilde{\Omega}_{1} t}+ \\
& +\left(B_{3} \sin \tilde{\Omega}_{2 d} t+B_{4} \cos \tilde{\Omega}_{2 d} t\right) e^{-\tilde{Z}_{2} \tilde{\Omega}_{2} t}+\frac{(1-\lambda) g}{2 \Omega_{y}^{2}} \\
x(t)= & \left(\frac{G \tilde{\Omega}_{1}^{2}}{\Omega_{x}^{2}-\tilde{\Omega}_{1}^{2}}\right)\left(B_{1} \sin \tilde{\Omega}_{1 d} t+B_{2} \cos \tilde{\Omega}_{1 d} t\right) e^{-\tilde{Z}_{1} \tilde{\Omega}_{1} t} \\
& +\left(\frac{G \tilde{\Omega}_{2}^{2}}{\Omega_{x}^{2}-\tilde{\Omega}_{2}^{2}}\right)\left(B_{3} \sin \tilde{\Omega}_{2 d} t+B_{4} \cos \tilde{\Omega}_{2 d} t\right) e^{-\tilde{Z}_{2} \tilde{\Omega}_{2} t}+\frac{G g}{\Omega_{x}{ }^{2}}
\end{aligned}
$$




$$
\begin{aligned}
& \tilde{\Omega}_{1,2}^{2}=\frac{\Omega_{x}{ }^{2}+\Omega_{y}{ }^{2} \mp \sqrt{\left(\Omega_{x}{ }^{2}-\Omega_{y}{ }^{2}\right)^{2}-4 \frac{\lambda G}{\alpha} \Omega_{x}{ }^{2} \Omega_{y}{ }^{2}}}{2\left(1-\frac{\lambda G}{\alpha}\right)} \\
& \tilde{Z}_{i}=\frac{Z_{x}\left(\frac{\tilde{\Omega}_{i}}{\Omega_{y}}-\frac{\Omega_{y}}{\tilde{\Omega}_{i}}\right)+Z_{y}\left(\frac{\tilde{\Omega}_{i}}{\Omega_{x}}-\frac{\Omega_{x}}{\tilde{\Omega}_{i}}\right)}{2\left(1-\frac{\lambda G}{\alpha}\right) \frac{\tilde{\Omega}_{i}^{2}}{\Omega_{x} \Omega_{y}}-\frac{\Omega_{x}}{\Omega_{y}}-\frac{\Omega_{y}}{\Omega_{x}}}, \quad i=1,2 \\
& \tilde{\Omega}_{i d}=\tilde{\Omega}_{i} \sqrt{1-\tilde{Z}_{i}^{2}}, \quad i=1,2 \\
& \Omega_{x}=\omega_{z} \sqrt{\frac{\alpha^{2}(1+\lambda)}{2\left[\alpha^{2}(1+\lambda)-\lambda\right]}} \\
& \Omega_{y}=\omega_{y} \quad \sqrt{\frac{1+\lambda}{2}} \\
& Z_{x}=\zeta_{x} \sqrt{\frac{\alpha^{2}(1+\lambda)}{2\left[\alpha^{2}(1+\lambda)-\lambda\right]}} \\
& Z_{y}=\zeta_{y} \sqrt{\frac{1+\lambda}{2}} \\
& G=\frac{\alpha \lambda}{\alpha^{2}(1+\lambda)-\lambda} \text {. }
\end{aligned}
$$

Again, equations (27) and (28) are correct to first order in $\zeta$ 's only. The rocking response can be found by double integration of equation (23), which gives

$$
h \varphi(t)=-\frac{\lambda}{\alpha^{2}(1+\lambda)} x(t) ;-\frac{\lambda}{\alpha(1+\lambda)} Y(t)-\frac{\lambda g}{2 \alpha(1+\lambda)} t^{2}+B_{5} t+B_{6}
$$

The coefficients $B_{1}$ to $B_{6}$ can be determined by the initial conditions, i.e., the final condition of the full-contact case.

Because of the parabolic terms which appear in equation (35), the system does not possess a natural rocking period, in the classical sense, after uplift. For the overall response, however, an apparent rocking period can be found from the time history of the motion. Since uplift results in a softer vibrating system, the apparent rocking frequency is expected to be smaller than $\omega_{\varphi}$, the natural frequency of rocking during full contact. Note that because of the nonlinearity of the problem, the apparent rocking period depends on the amplitude of the response.

It is interesting to note that since $\alpha$ and $\lambda$ can be written as functions of the ratios $b / a, h / b$, and $\xi / a$ only, the value of the product $h \varphi$, which denotes the 
horizontal displacement of the center of mass due to the rotation, is independent of the exact dimensions of the block and is only affected by these ratios (see the equations of motion). In other words, the value of $h \varphi(t)$, for a certain initial velocity $v_{0}$, will be the same for two blocks of different size, but of the same ratios $b / a, h / b$, and $\xi / a$, provided that $\omega_{x}, \omega_{y}, \zeta_{x}$ and $\zeta_{y}$ are the same. The latter condition implies that, for the same stiffness of the springs in the two cases, the densities of the blocks should be different in order for the masses to be equal. As a result of these factors, the same initial impulse will cause different angles of rotation for different size blocks of the same mass, in order for the product $h \varphi$ to be the same; the angle $\varphi$ will be larger for smaller blocks. This size effect may be important in practical cases, because the possibility of overturning is associated with the angle of rotation.

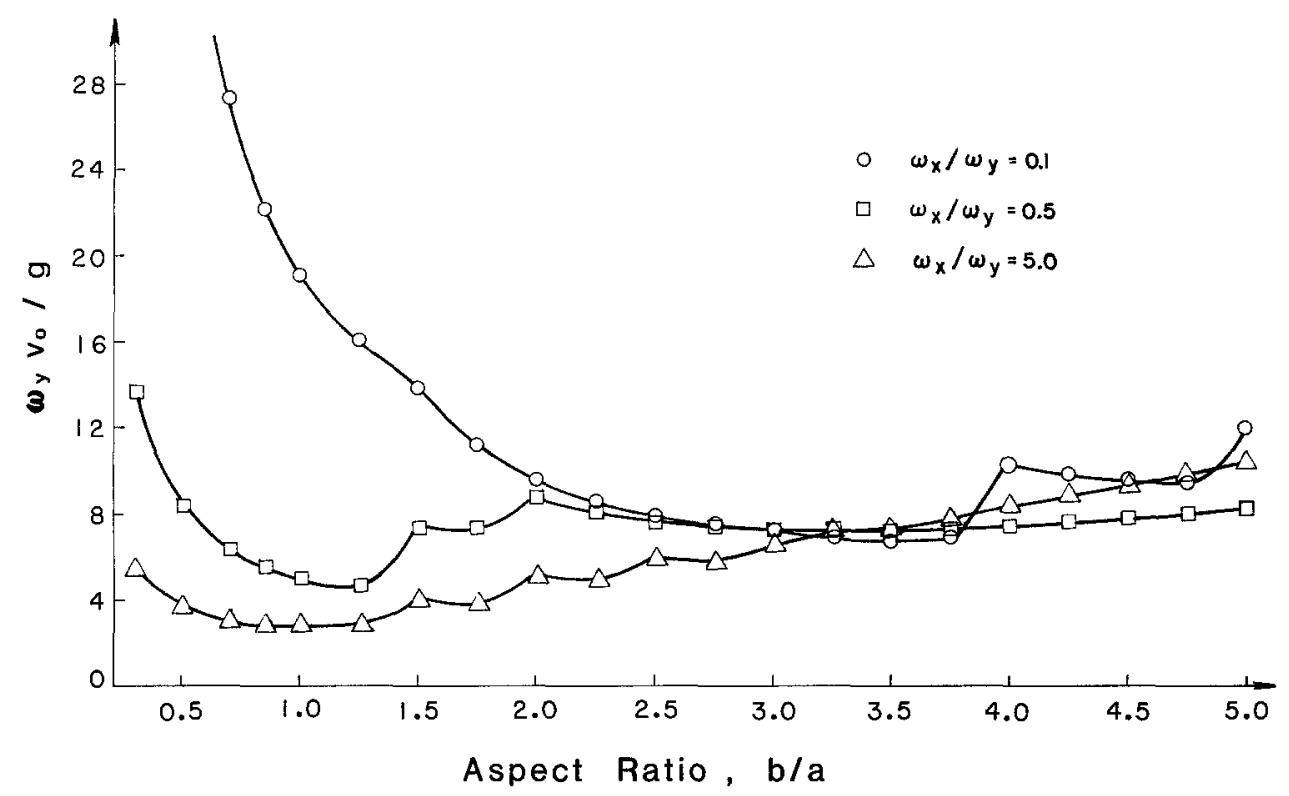

FIG. 2. Dependence of the minimum required value of $\omega_{y} v_{0} / g$ for upthrow on the aspect ratio $b / a\left(\zeta_{x}=\zeta_{y}=10\right.$ per cent $)$.

If the excitation is not strong enough to cause complete separation, the system will vibrate, changing between the two regimes of full contact and partial uplift. For strong excitations, however, upthrow is possible. Since $Y$ denotes the vertical displacement of the point of contact, complete separation will happen if

$$
Y<-\delta \text {. }
$$

The amplitude of $Y$ depends on the initial horizontal velocity, $v_{0}$, and on the parameters of the system. It was found in the analysis that even for high values of damping, complete separation does not necessarily occur during the first half rocking period, but it may happen during the second or even the third half period. Because of this observation and the coupling between the three modes of vibration and the two regimes of the response, an analytical expression for a criterion for the occurrence of upthrow appears to be a practical impossibility. In the following section, several particular cases are solved and the minimum required value of $v_{0}$ for upthrow is determined, in an effort to understand the importance of the parameters upon this phenomenon. 


\section{PARAMETRIC INVESTIGATION}

All the analysis in this section is done for the case of a homogeneous block ( $h=b / 2)$ supported by springs placed at the corners of the base $(\zeta=a / 2)$. The equations of motion were solved numerically using the Runge-Kutta-Gill method with a time step of $0.001 \mathrm{sec}$. The minimum value of the initial horizontal velocity, $v_{0}$, required for complete separation was determined by trial and error. Instead of the absolute magnitude of $v_{0}$, however, the dimensionless quantity $\omega_{y} v_{0} / g$ is used as a measure of the impulse excitation. Since the potential energy initially stored in the springs is

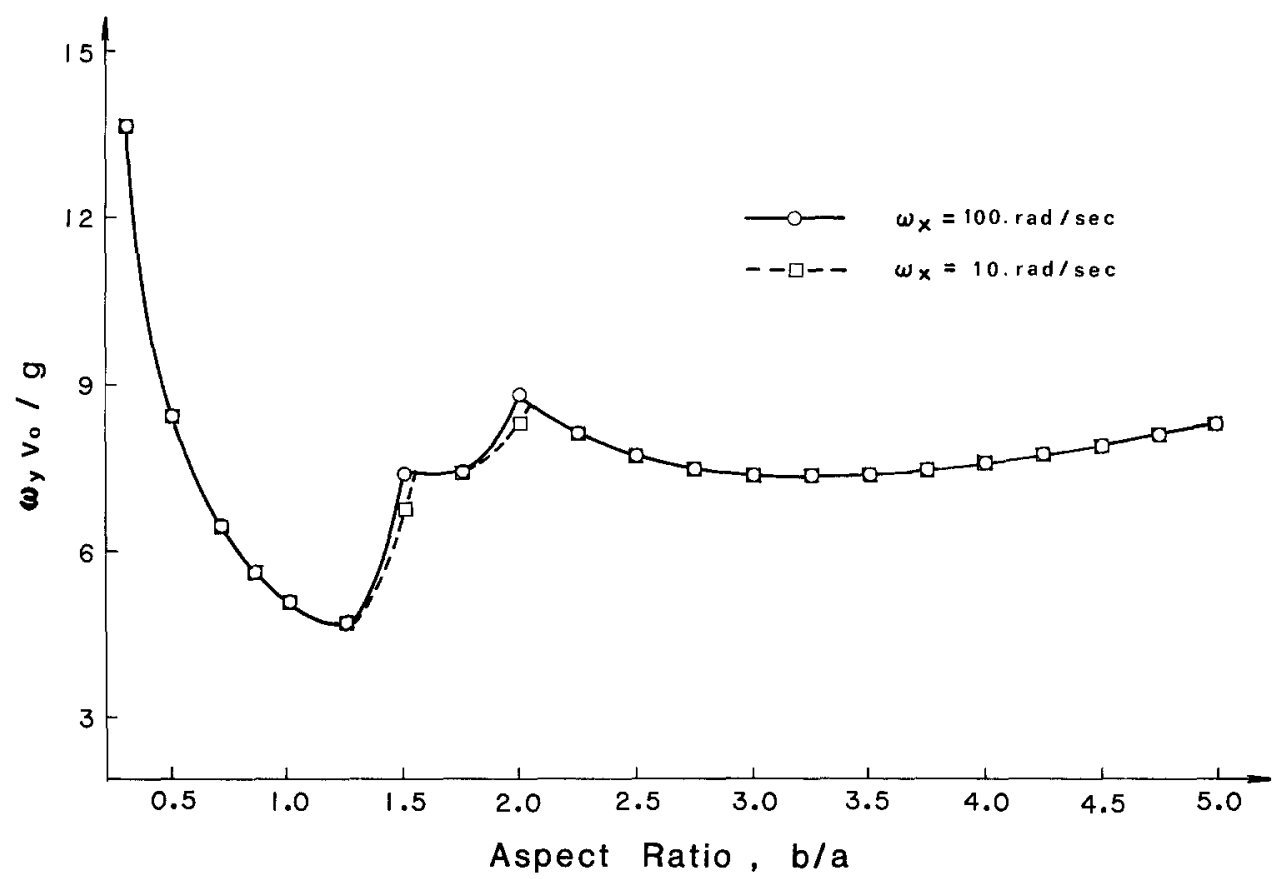

FIG. 3. Illustration of the independence of the minimum required value of $\omega_{y} v_{0} / g$ for upthrow on the particular value of $\omega_{x}$ or $\omega_{y}\left(\omega_{x} / \omega_{y}=0.5, \zeta_{x}=\zeta_{y}=10\right.$ per cent $)$.

$$
P E=\frac{1}{2}\left(2 k_{y}\right) \delta^{2}=\frac{1}{2} m \frac{g^{2}}{\omega_{y}{ }^{2}}
$$

and the kinetic energy introduced by the impulse is

$$
K E=\frac{1}{2} m v_{0}^{2},
$$

the normalized impulse is equal to

$$
\frac{\omega_{y} v_{0}}{g}=\sqrt{\frac{K E}{P E}}
$$

In Figure 2, the minimum required value of $\omega_{y} v_{0} / g$ for upthrow is plotted versus the aspect ratio $b / a$ for three different values of the ratio $\omega_{x} / \omega_{y}$. It should be noted that this plot is independent of the particular value of $\omega_{x}$ or $\omega_{y}$, but it only depends on the ratio $\omega_{x} / \omega_{y}$. This property, which can be established analytically, is illustrated in Figure 3, where the plot for $\omega_{x} / \omega_{y}=0.5$ is shown for $\omega_{x}=100 \mathrm{rad} / \mathrm{sec}$ and $\omega_{x}=$ 
$10 \mathrm{rad} / \mathrm{sec}$. Except for small differences around the peaks, which are due to the numerics, it can be said that the two curves coincide.

As it is evident from Figure 2, a very strong impulse is required for complete separation of short blocks and relatively soft horizontal springs. This behavior was expected since most of the energy in this case goes into horizontal vibrations and the rocking amplitude is small. As the aspect ratio $b / a$ increases, however, it seems that the relative stiffness of the springs is not so important and all three curves are close to each other.

The kinks that appear on the curves in Figures 2 and 3 are difficult to explain physically. It is evident that the angular velocity at the time of partial separation, which depends on $\tilde{\omega}_{1}$ and $\tilde{\omega}_{2}$, plays an important role in determining whether complete separation will occur or not. In most cases, complete separation occurs in the beginning of the second half rocking period and in this case, the angular and

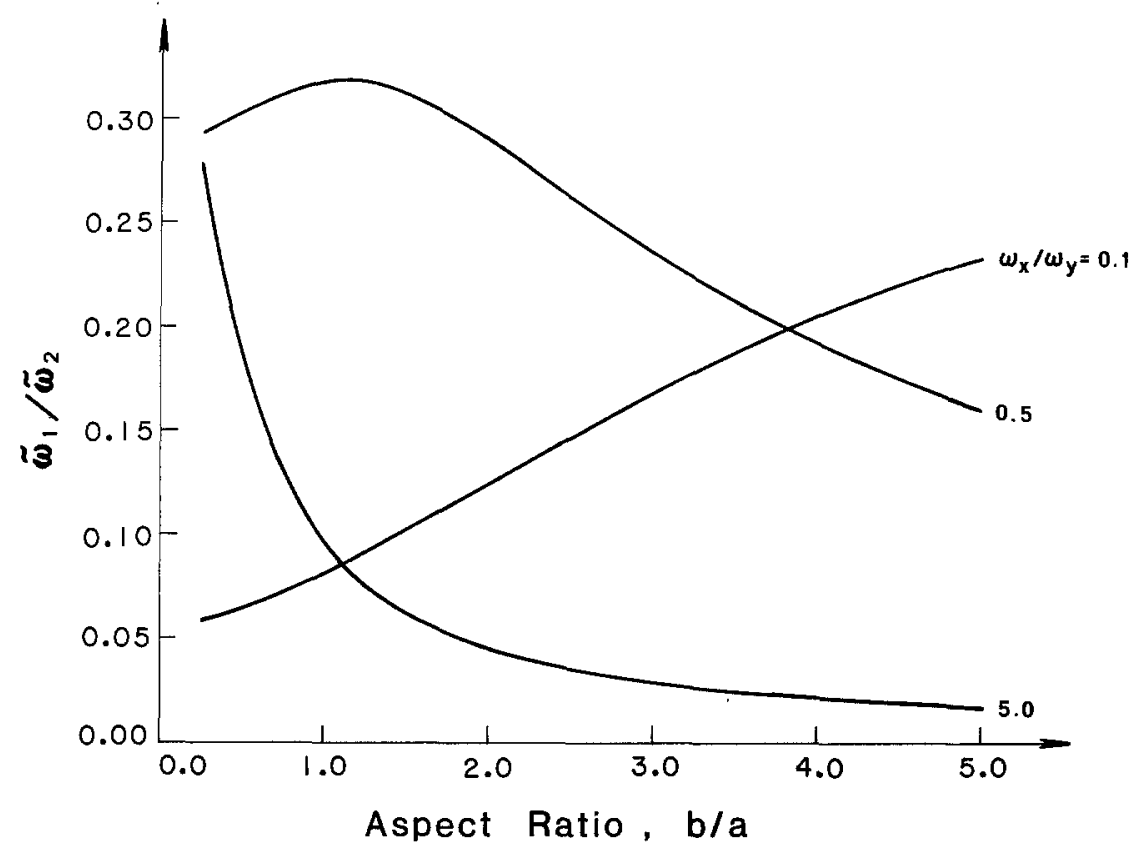

FiG. 4. Variation of the ratio $\tilde{\omega}_{1} / \tilde{\omega}_{2}$ with the aspect ratio $b / a$ and the frequency ratio $\omega_{x} / \omega_{y}$.

vertical velocities at the time when full contact is reestabished are very important, too. It appears that small changes in the parameters of the system may affect significantly these velocities in some cases, and as a result, large changes in the minimum required value of $\omega_{y} v_{0} / g$ appear, which cause the kinks in Figure 2 . The relative values of $\tilde{\omega}_{1}$ and $\tilde{\omega}_{2}$, and of $\tilde{\Omega}_{1}$ and $\tilde{\Omega}_{2}$ are also important parameters in the behavior. In Figure 4, the ratio $\tilde{\omega}_{1} / \tilde{\omega}_{2}$ is plotted versus $b / a$ for the three cases of $\omega_{x} / \omega_{y}=0.1,0.5$, and 5.0. It is noted that the valley which appears in Figure 2 for $b / a=1.25$ and $\omega_{x} / \omega_{y}=0.5$ can be associated with the peak of the corresponding curve in Figure 4.

Although the response to an impulse cannot be applied directly to the case of earthquake excitation, the results can provide some information in the case of strong earthquakes containing large pulses. Even in that case, however, one should be careful, because earthquake pulses occur over a relatively long time compared to 
the characteristic small-amplitude periods of the systems expected to be encountered in practice, while the previous analysis was based on the assumption of an instantaneous impulse. For example, the large pulse in the Pacoima Dam record from the San Fernando earthquake has a duration of about $0.65 \mathrm{sec}$. For the results of the present study to be applicable to objects or structures excited by such pulses, the response to the pulse must be large enough that the effective, large-amplitude rocking period of the system is around $2 \mathrm{sec}$ or longer. With this limitation, the plots in Figure 2 can be used to suggest whether or not horizontal ground motion during an earthquake might have been sufficient to cause upthrow.

As an example, take $\omega_{y}=100 \mathrm{rad} / \mathrm{sec}(\approx 16 \mathrm{~Hz})$. Then, a sharp pulse of $v_{0}=0.525 \mathrm{~m} / \mathrm{sec}$. which is equal to the maximum pulse in the S00E component of

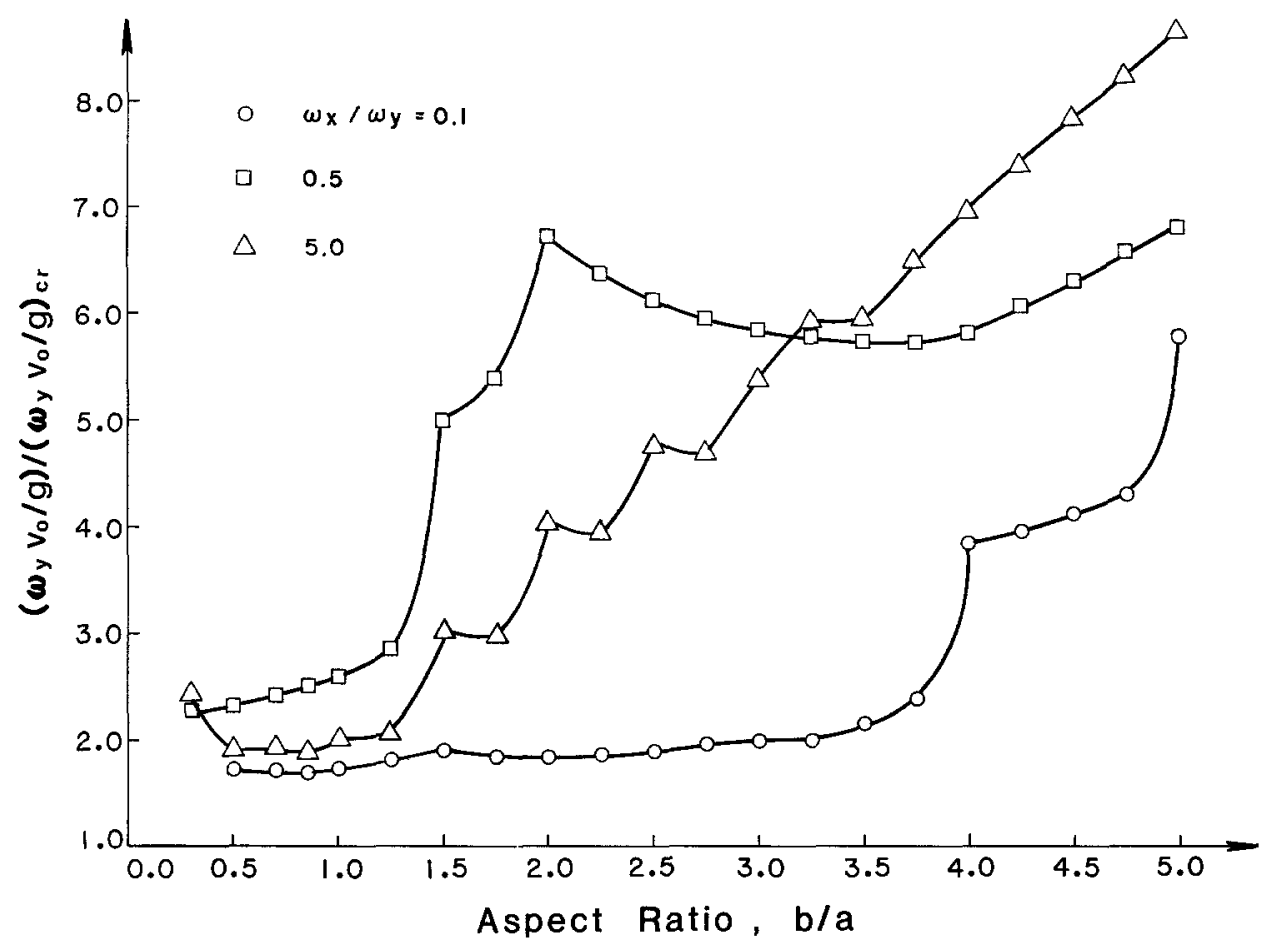

FIG. 5. Variation of the minimum required value of $\omega_{y} v_{0} / g$ for upthrow with the aspect ratio $b / a\left(\zeta_{x}=\zeta_{y}=10\right.$ per cent $)$.

the El Centro earthquake of 18 May 1940 (Housner, 1978), would produce a value of $\omega_{y} v_{0} / g$ equal to 5.35 , which is adequate to cause complete separation in the case of short blocks with stiff horizontal springs. Similarly, a sharp pulse equal in magnitude to the large pulse recorded at the Pacoima Dam would produce a value of $\omega_{y} v_{0} / g$ around 15 , large enough to cause upthrow in most cases. The additional consideration of vertical ground motion is believed to increase the possibility of upthrow, since vertical excitation alone is capable of causing complete separation in some cases (e.g., Newmark, 1973).

In Figure 5 , the required value of $\omega_{y} v_{0} / g$ for complete separation is compared with the critical value, $\left(\omega_{y} v_{0} / g\right)_{c r}$, required for partial uplift to occur. The ratio of these two numbers indicates how much stronger the excitation must be to cause total, rather than partial, uplift. Although the irregularity of the curves does not allow 
many general conclusions to be drawn, it seems that for stiff horizontal springs, the ratio $\left(\omega_{y} v_{0} / g\right) /\left(\omega_{y} v_{0} / g\right)_{c r}$ tends to be proportional to the aspect ratio $b / a$, at least for large values of $b / a$. On the contrary, for soft horizontal springs and less slender blocks, this ratio seems to be independent of the ratio $b / a$.

The dependence of the minimum required value of $\omega_{y} v_{0} / g$ on the frequency ratio $\omega_{x} / \omega_{y}$ is shown in Figure 6 for $b / a=2$ and several values of the damping coefficients. Again, these plots depend only on the value of the ratio $\omega_{x} / \omega_{y}$ and not on the particular value of $\omega_{x}$ or $\omega_{y}$. It is interesting to note that the local peak around $\omega_{x} /$ $\omega_{y}=0.5$, which appears in all these curves, can be associated with the peak that appears in the plot of $\tilde{\omega}_{1} / \tilde{\omega}_{2}$ versus $\omega_{x} / \omega_{y}$, shown in Figure 7 . This is another indication that the relative value of the natural frequencies $\tilde{\omega}_{1}$ and $\tilde{\omega}_{2}$ is important for upthrow.

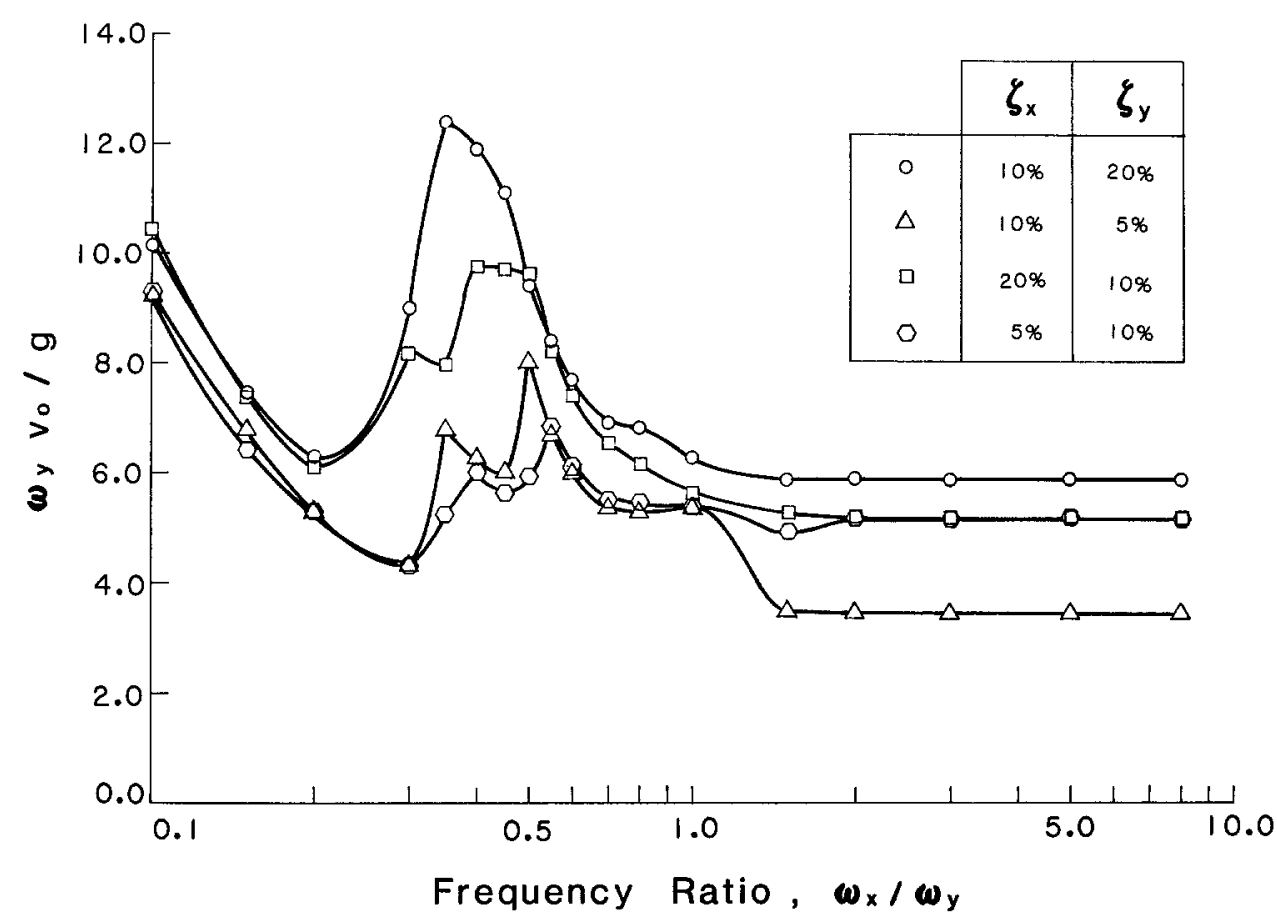

FIG. 6. Dependence of the minimum required value of $\omega_{y} v_{0} / g$ for upthrow on the frequency ratio $\omega_{x} / \omega_{y}$ and the foundation damping $(b / a=2)$.

An interesting result can be drawn from Figure 6 concerning the effect of the damping. It is evident that a small increase in the damping coefficients in either the horizontal or vertical directions increases significantly the required initial impulses for upthrow. In some cases, an increase of the damping coefficient from 5 to 20 per cent can almost double the required value of $\omega_{y} v_{0} / g$ for complete separation. It appears, therefore, that foundation damping may play a controlling role in the possible occurrence of upthrow in practical cases.

\section{EXAMPLES}

The time history of the response for $\omega_{x} / \omega_{y}=0.5, \omega_{y} v_{0} / g=5$ and two values of $b / a$, namely 1.25 and 1.5 , is shown in Figures 8 to 11 . Note that this value of $\omega_{y} v_{0} / g$ is strong enough to cause upthrow for $b / a=1.25$ but not for $b / a=1.5$. In 
this example, $\zeta_{x}=\zeta_{y}=10$ per cent and $\omega_{z}=100 \mathrm{rad} / \mathrm{sec}$. For $b / a=1.25$, complete separation occurs in the beginning of the second half rocking period. When full contact is reestablished after the first half-cycle, the rocking and vertical velocities are so large that they force the left corner of the base to move downwards, a distance equal to almost four times the static deflection, $\delta$ (see Figure 10). This gives the left vertical spring enough potential energy to upthrow the block a little later. In the case of $b / a=1.5$, although the rocking velocity at the time of recontact is about the same as for $b / a=1.25$, the downward vertical velocity of the right corner is much smaller and as a result, the left corner moves downwards less than three times the static deflection, not enough to lead to complete separation. A much larger value of $\omega_{y} v_{0} / g$ (7.32) is required for upthrow in this case. It is this phenomenon that is believed to be responsible for the kinks, such as seen in Figures 2 and 3.

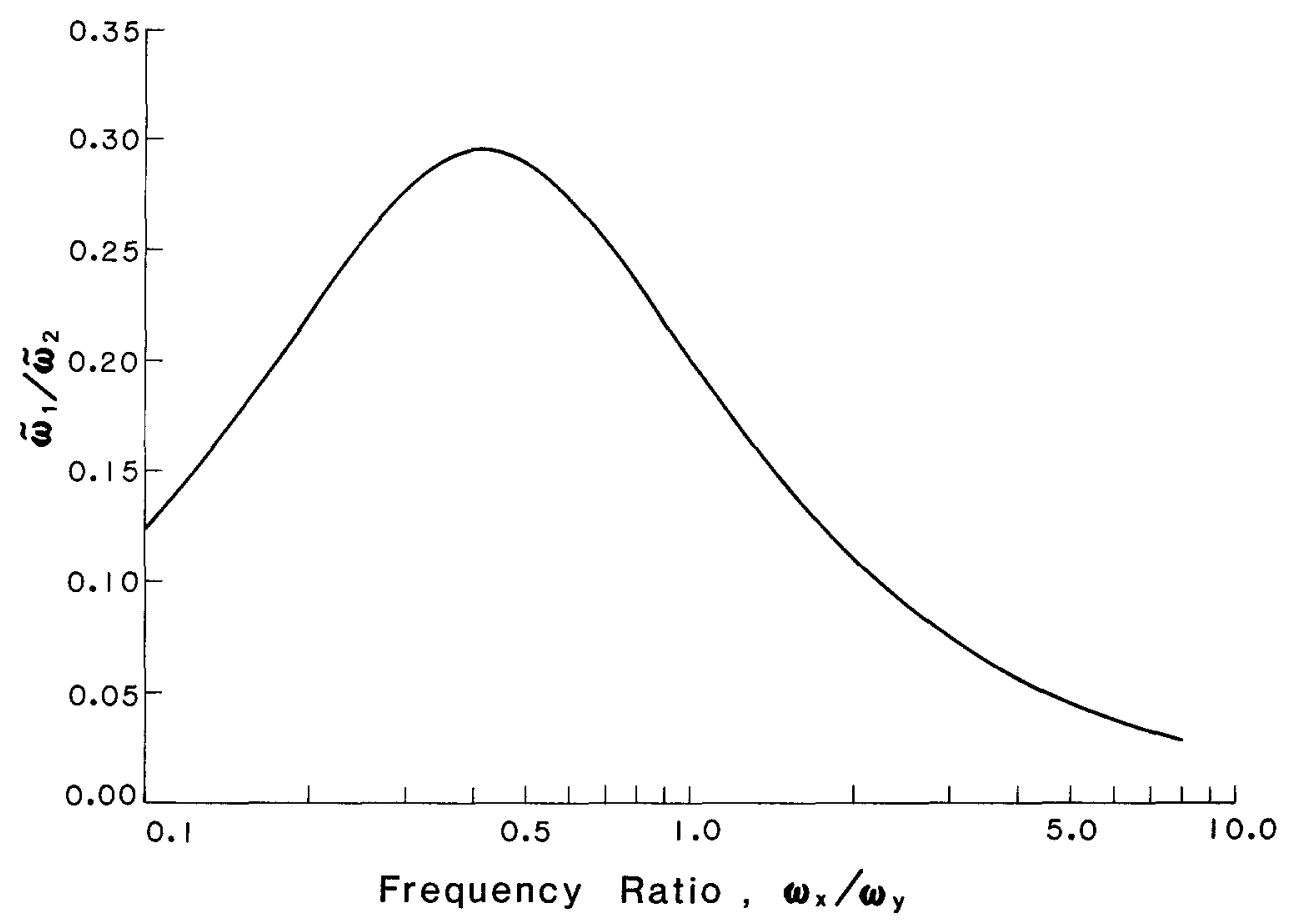

FIG. 7. Variation of the ratio $\tilde{\omega}_{1} / \tilde{\omega}_{2}$ with the frequency ratio $\omega_{x} / \omega_{y}$ for $b / a=2$.

Although, in most instances, the minimum value of $\omega_{y} v_{0} / g$ caused complete separation during the second half period, upthrow will occur during the first half period for very strong excitations. This is illustrated in Figure 11, where the vertical displacement of the right corner of the block is shown. In this case, the block almost separates completely at $0.06 \mathrm{sec}$. It is evident that for stronger excitation the amplitude of the vertical vibrations will increase and complete separation will occur before full contact is reestablished.

After complete separation, the block continues to move under its weight and eventually it will regain contact with the ground. The animated motion of the block until that time is shown in Figures 12 to 14 for three examples with $\omega_{y} v_{0} / g=15$. Because of the large values of the angle $\varphi$ involved in these examples, the full $\sin \varphi$ and $\cos \varphi$ terms were used in the equations of motion after complete separation, rather than the small-angle approximations. The effect of the actual size of the 
block on the angle of rotation (discussed earlier) is illustrated in Figures 13 and 14; the aspect ratio $b / a$ and the values of $\omega$ 's and $\zeta$ 's are the same in these two examples, but the size of the block in Figure 13 is one third the size of the one in Figure 14. It is evident that the angle of rotation is much larger for the smaller block and that complete overturning will result. In contrast, the bigger block has a more stable behavior.

\section{Discussion}

This study is essentially a brief parametric investigation of the upthrow of objects, excited into motion by a horizontal impulse. In spite of its seeming simplicity, the

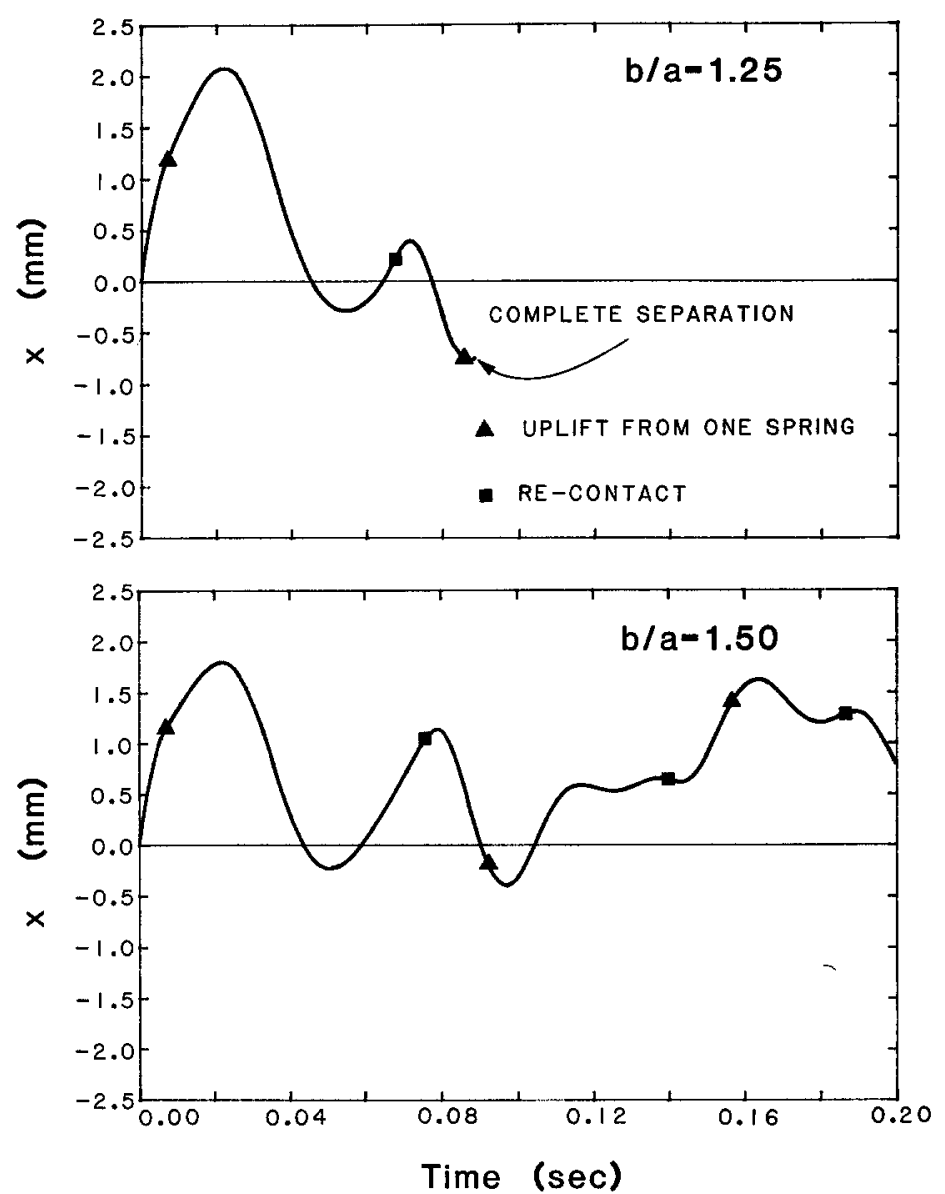

FIG. 8. Horizontal displacement of the mid-point of the base, $M$, for $\omega_{x} / \omega_{y}=0.5, \omega_{x}=100 \mathrm{rad} / \mathrm{sec}$, $\zeta_{x}=\zeta_{y}=10$ per cent and $\omega_{y} v_{0} / g=5$.

problem of a rocking rigid block that can separate from its foundation is nonlinear and very complicated analytically. Thus, explicit formulas which govern the phenomenon of complete separation were not found. In this analysis, the minimum required value of the horizontal impulse for upthrow to occur was determined numerically for a number of examples. Analytical expressions for the solution of the equations of motion were also given. The dimensionless quantity $\omega_{y} v_{0} / g$ was 
used to measure the strength of the excitation; this can also be viewed as a measure of the initial kinetic energy imparted to the system.

The equation of motion and the example calculation confirm an effect of size in this problem. This effect has been noted by other authors and is supported by observations of the effects of earthquakes. In this study, the size effect is seen in the fact that for two uniform blocks, with the same mass, aspect ratio and foundation springs and dashpots, the same value of $h \varphi(t)$ results. This means that the smaller of the two blocks will rotate more, as is illustrated by Figures 13 and 14.
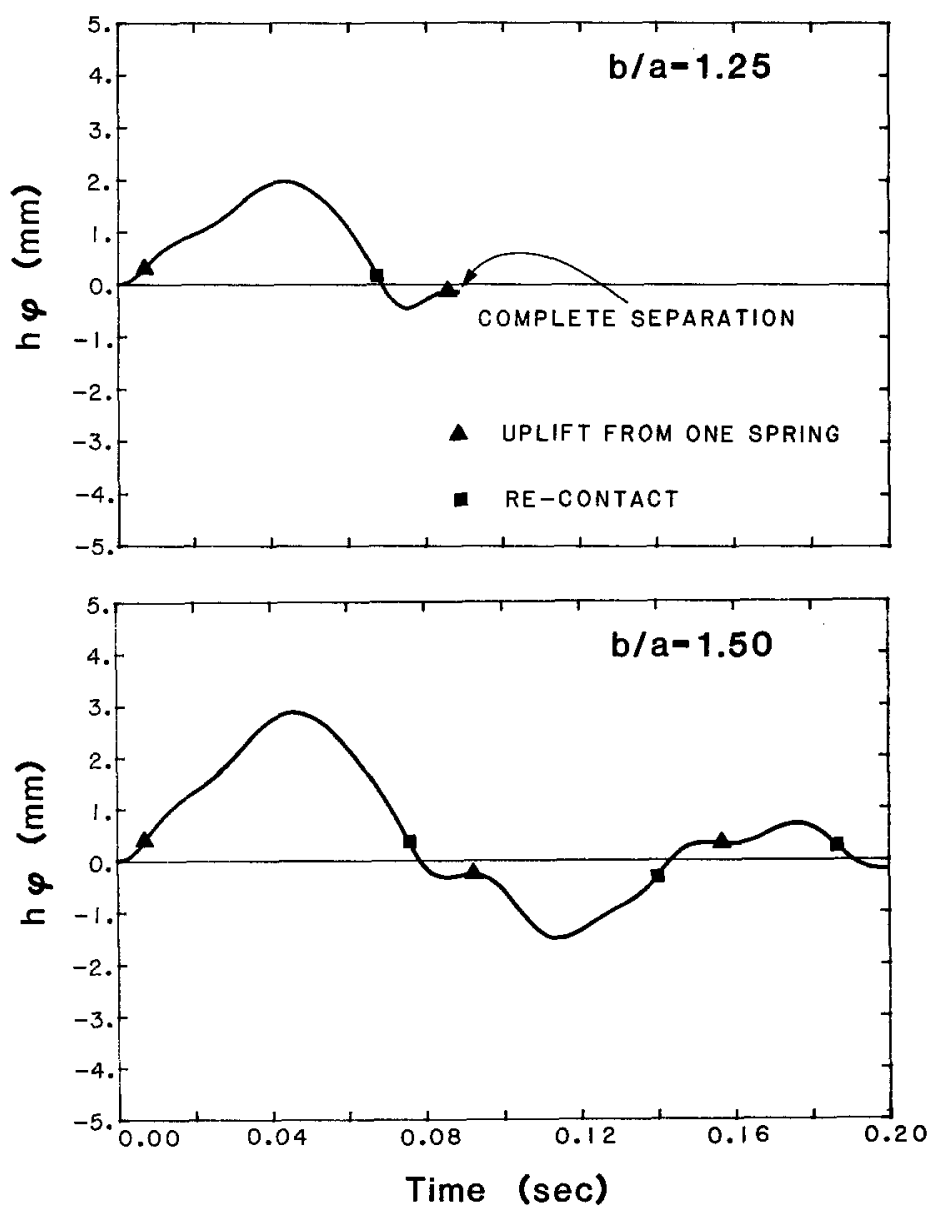

Fig. 9. Horizontal displacement of the center of mass due to rotation for $\omega_{x} / \omega_{y}=0.5, \omega_{x}=100 \mathrm{rad} /$ sec, $\zeta_{x}=\zeta_{y}=10$ per cent and $\omega_{y} v_{0} / g=5$.

The results illustrate many complexities which are difficult to explain; therefore, it is hard to draw general conclusions. It can be said that for short blocks, the required value of the initial impulse for complete separation increases as the stiffness of the horizontal springs decreases and as the aspect ratio $b / a$ decreases. For tall blocks, however, the dependence of upthrow on the relative stiffness of the vertical and horizontal springs and the aspect ratio of the block is not clear, and the results are mixed. If the minimum required impulse for upthrow is compared to the critical value required for partial uplift, the results indicate that the ratio of these quantities 
tends to be proportional to the aspect ratio $b / a$ for tall blocks and stiff horizontal springs. It also appears that the impulse required for separation is independent of the particular values of $\omega_{x}$ and $\omega_{y}$ and depends only on their ratio. Finally, the results suggest that foundation damping is very important and higher values of effective damping may prevent complete separation in practical cases.

For most of the cases examined, complete separation first happened during the second half rocking period. That is, the block rotates in the direction of the initial velocity, partially separating from the foundation. It then comes back through the
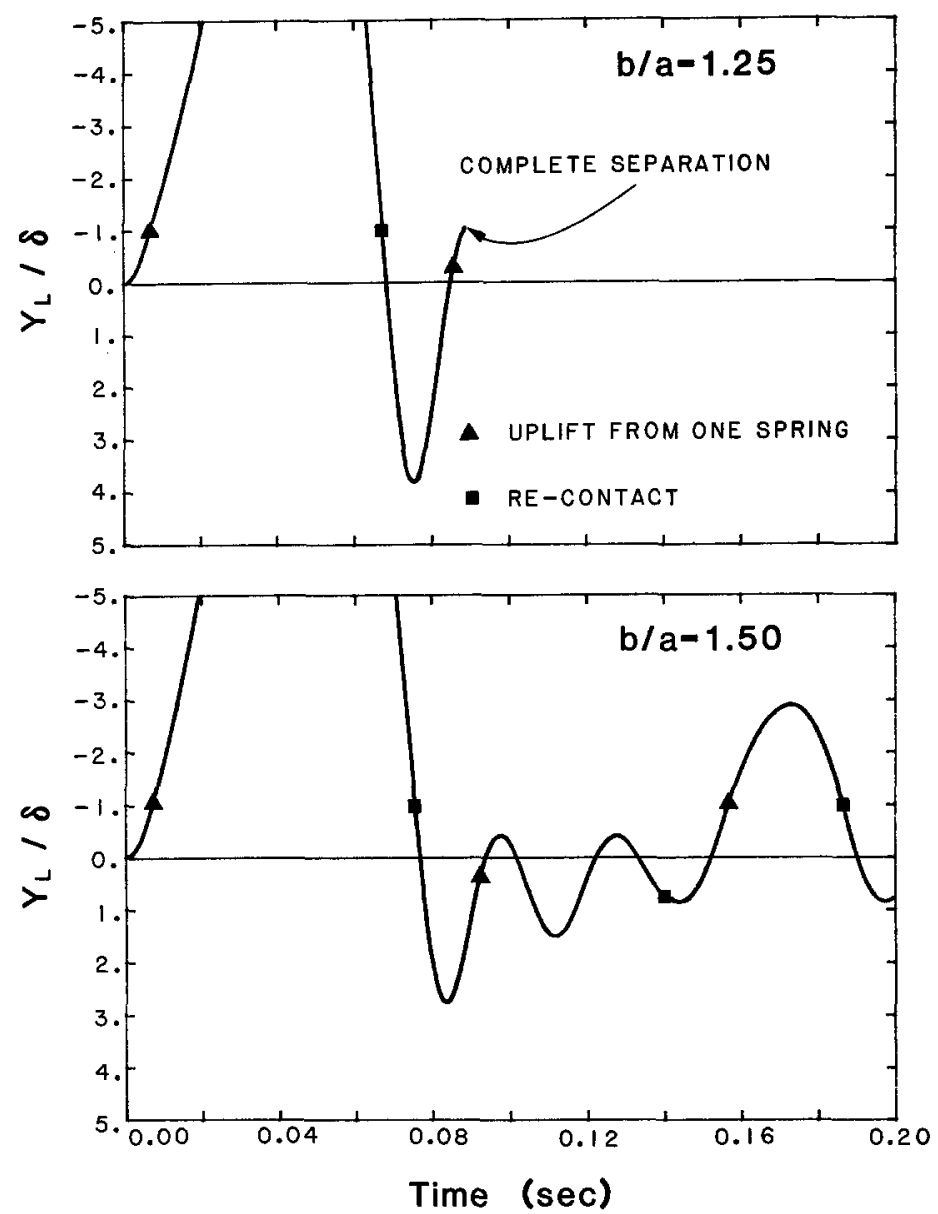

FIG. 10. Normalized vertical displacement of the left corner of the base for $\omega_{x} / \omega_{y}=0.5, \omega_{x}=100 \mathrm{rad} /$ sec, $\zeta_{x}=\zeta_{y}=10$ per cent and $\omega_{y} v_{0} / g=5$.

vertical position, the other corner then penetrates deeply and then the block is upthrown. For larger excitations, however, upthrow occurs during the first half period. In this case, the block's corner penetrates the foundation so deeply on the first half cycle that it is upthrown before it regains the vertical position (see Figures 12 to 14). It should be noted that upthrow of an object does not necessarily imply that overturning will also occur; this depends on the magnitude of the impulse, the stiffness of the foundation and the dimensions of the block.

Although the results of the impulse response cannot be applied directly to the 

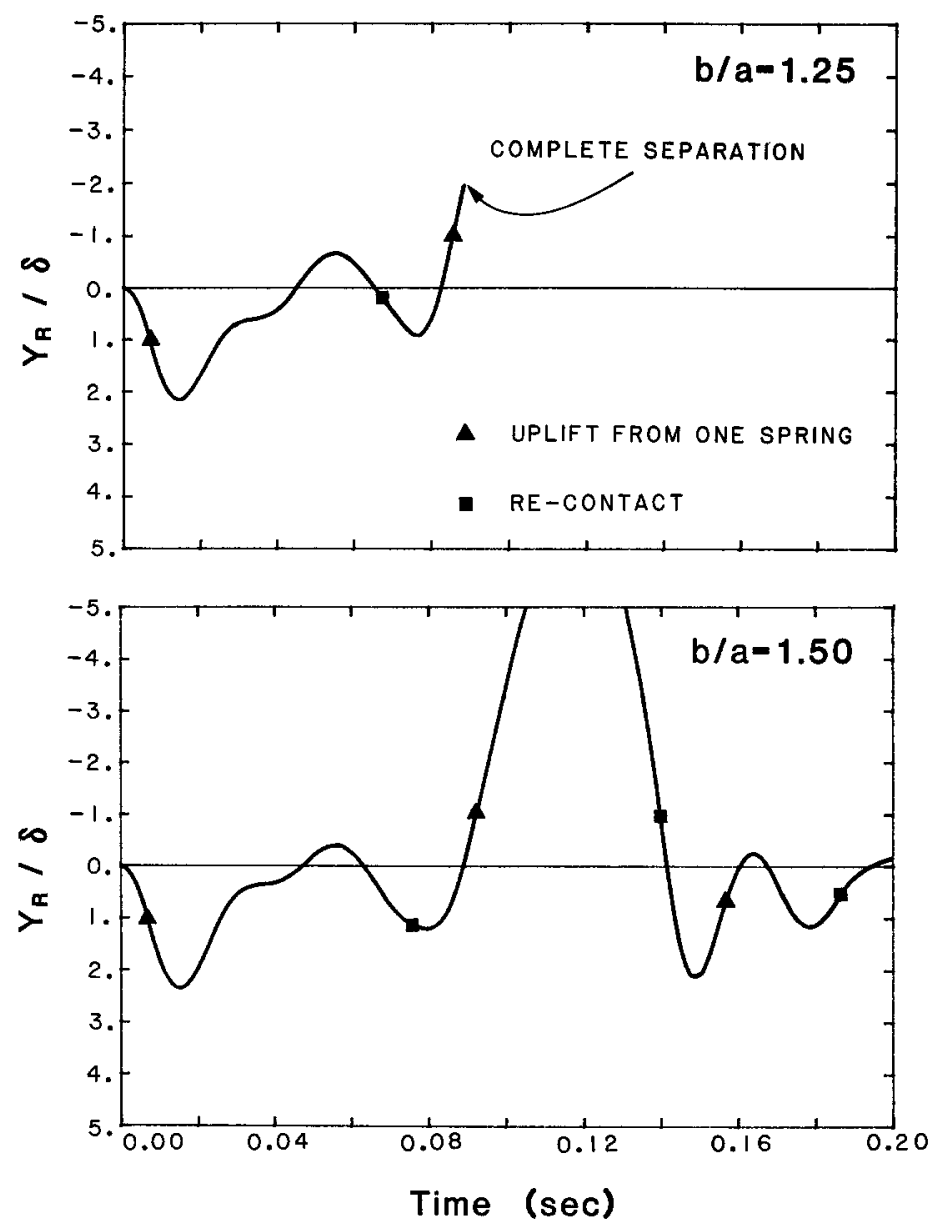

FIG. 11. Normalized vertical displacement of the right corner of the base for $\omega_{x} / \omega_{y}=0.5, \omega_{x}=100$ $\mathrm{rad} / \mathrm{sec}, \zeta_{x}=\zeta_{y}=10$ per cent and $\omega_{y} v_{0} / g=5$.

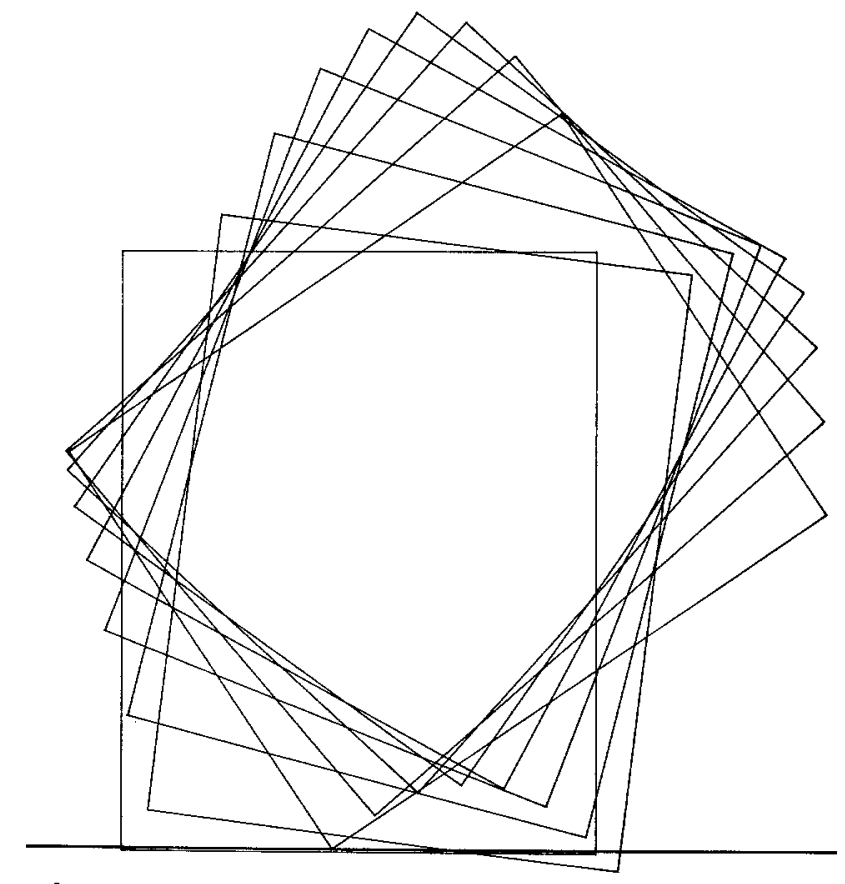

Fig. 12. Animated motion of the upthrown block for $a=0.5 \mathrm{~m}, b / a=1.25, \omega_{x}=50 \mathrm{rad} / \mathrm{sec}, \omega_{y}=65$ $\mathrm{rad} / \mathrm{sec}, \zeta_{x}=\zeta_{y}=10$ per cent and $\omega_{y} v_{0} / g=15$. 


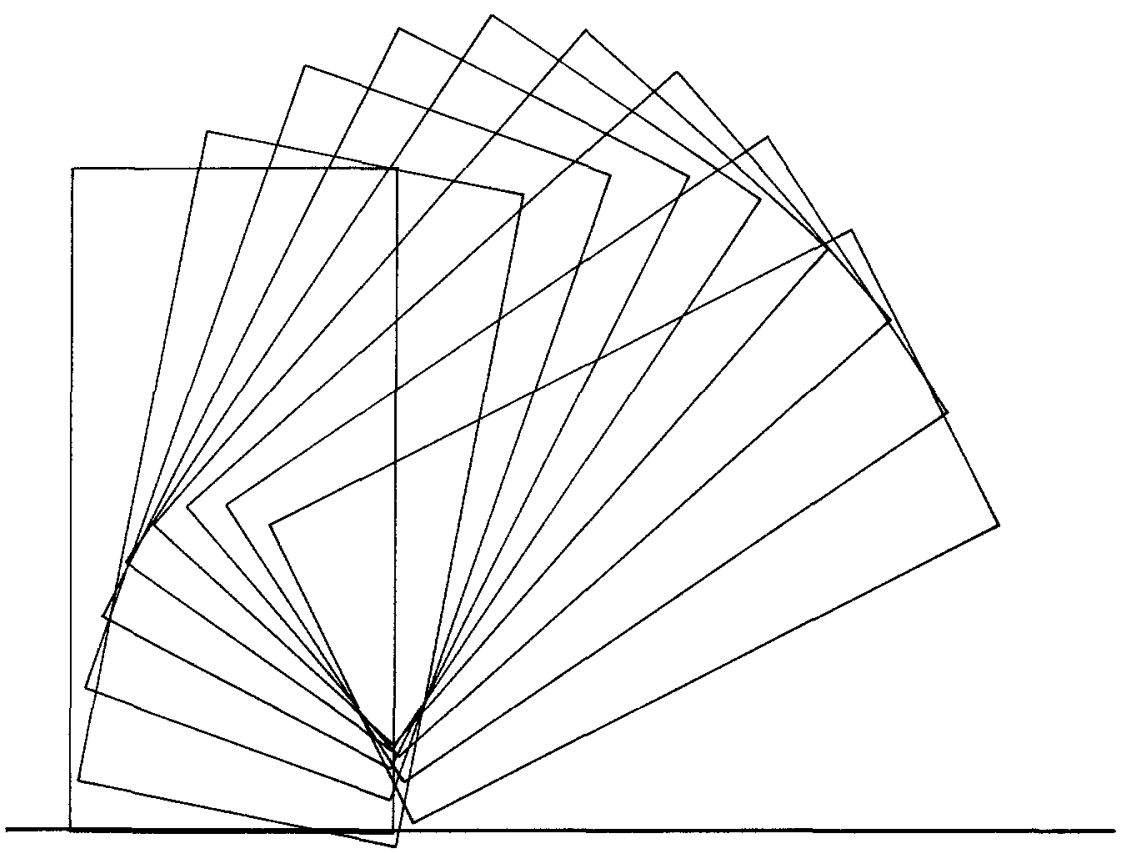

FIG. 13. Animated motion of the upthrown block for $a=0.5 \mathrm{~m}, b / a=2, \omega_{x}=100 \mathrm{rad} / \mathrm{sec}, \omega_{y}=50$ $\mathrm{rad} / \mathrm{sec}, \zeta_{x}=\zeta_{y}=10$ per cent and $\omega_{y} v_{0} / g=15$.

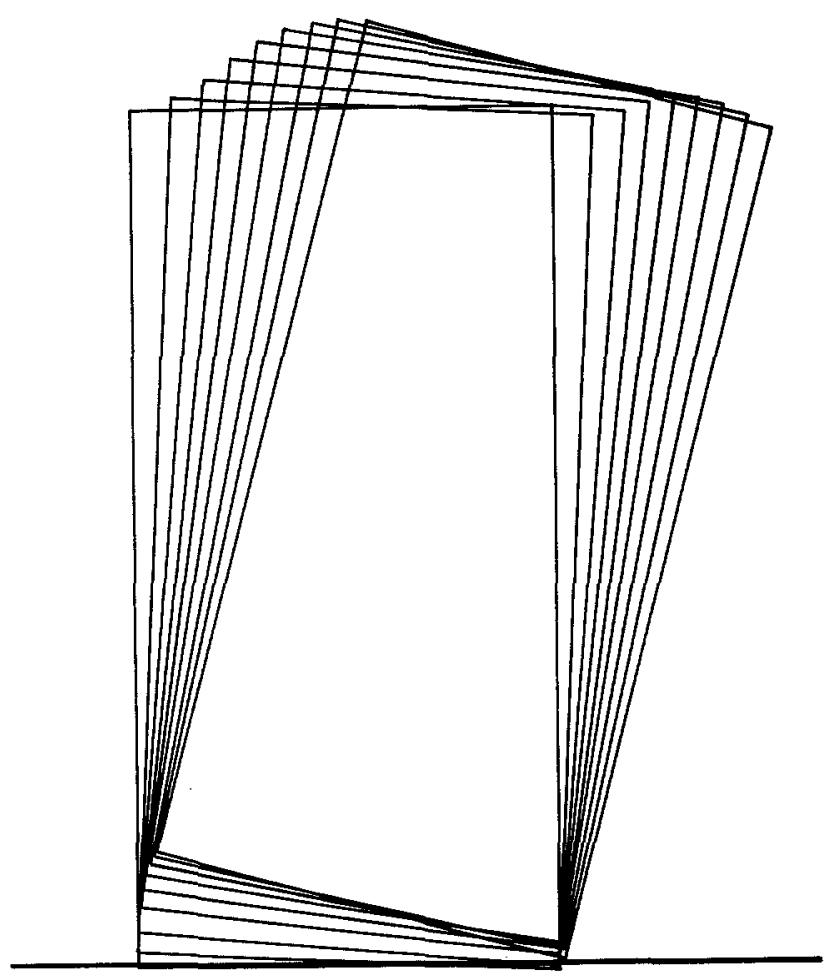

Fig. 14. Animated motion of the upthrown block for $a=1.5 \mathrm{~m}, b / a=2, \omega_{x}=100 \mathrm{rad} / \mathrm{sec}, \omega_{y}=50$ $\mathrm{rad} / \mathrm{sec}, \zeta_{x}=\zeta_{y}=10$ per cent and $\omega_{y} v_{0} / g=15$. 
case of earthquake excitation, they can provide insight in some cases. Application of these results to two earthquake records which contain large pulses indicates that the horizontal component of these motions may be adequate to cause upthrow of some objects. Because complete separation of this system is clearly possible under vertical excitation only, the possibility of upthrow is thought to be higher when vertical excitation is also present.

\section{REFERENCES}

Bolt, B. A. and R. A. Hansen (1977). The upthrow of objects in earthquakes, Bull. Seism. Soc. Am. 67, 1415-1427.

Clark, M. M. (1972). Intensity of shaking estimated from displaced stones, in The Borrego Mountain Earthquake of April 9, 1968, U.S. Geol. Surv. Profess. Paper 787, 175-182.

Housner, G. W. (1978). Statistics of pulses on strong motion accelerograms, Proceedings of the N.S.F. Seminar-Workshop on Strong Ground Motion, Rancho Santa Fe, Santa Fe, New Mexico, 60-64.

Ikegami, R. and F. Kishinouye (1950). The Acceleration of earthquakes motion deduced from overturning of the gravestones in case of the Imaichi earthquake on December 26, 1949, Bull. Earthquake Res. Inst., Tokyo Univ. 28, 121-128.

Ishiyama, Y. (1960). Review and discussion on overturning of bodies by earthquake motions, BRI Research Paper No. 85, Building Research Institute, Ministry of Construction.

Ishiyama, Y. (1982). Motion of rigid bodies and criteria for overturning by earthquake excitations. Earthquake Eng. Struct. Dyn. 10, 635-650.

Kawasumi, H. (1950). The Imaichi Earthquake of December 26, 1949-General Description. Bull. Earthquake Res. Inst., Tokyo Univ. 28, 355-367.

Lawson, A. C. (Editor) (1908). The California Earthquake of April 18, 1906, Report of State Earthquake Investigation Commission, Carnegie Institution, p. 201.

Matsuzawa, T. (1944). Über die Verschiebung von Komainu bei dem Tottori-Grossbeben, Bull. Earthquake Res. Inst., Tokyo Univ. 22, 60-65.

Morrill, B. J. (1971). Evidence of record vertical accelerations at Kagel Canyon during the earthquake, The San Fernando, California, Earthquake of February 9, 1971, U.S. Geological Survey and National Oceanic and Atmospheric Administration, U.S. Geol. Surv. Profess. Paper 733, 177-181.

Newmark, N. M. (1973). Interpretation of Apparent Upthrow of Objects in Earthquakes, Fifth World Conference on Earthquake Engineering, Rome, Italy, Paper 294, 2338-2343.

Oldham, R. D. (1899). Report on the Great Earthquake of 12th June 1897, Memoirs of the Geological Survey of India, Geological Survey of India, vol. XXIX, Calcutta, India.

Psycharis, I. N. and P. C. Jennings (1982). Rocking of slender rigid bodies allowed to uplift. Earthquake Eng. Struct. Dyn. 11, 57-76.

Richter, C. F. (1958). Elementary Seismology, W. H. Freeman and Co., San Francisco, California, 497499.

Division of ENGINeERING AND APPLIEd ScIENCE

CALIFORNia INSTITUTE OF TECHNOLOGY

Pasadena, California 91125

Manuscript received 22 November 1983 